\title{
A new fractal dimension definition based on intuitionistic fuzzy logic
}

\section{Oscar Castillo and Patricia Melin}

\author{
Division of Graduate Studies and Research \\ Tijuana Institute of Technology \\ Tijuana, Mexico \\ e-mails: ocastillo@tectijuana.mx, pmelin@tectijuana.mx
}

Received: 4 March 2019

Revised: 20 April 2019

Accepted: 30 April 2019

\begin{abstract}
In this paper we describe a method for the estimation of the fractal dimension of a geometrical object using Intuitionistic fuzzy logic techniques. The mathematical concept of fractal dimension serves to measure the geometrical complexity of an object. The algorithms for estimating the fractal dimension compute a numerical value using as input the time series data for a particular problem. The result is a crisp value (number) that defines the complexity of the geometrical object or the time series. The estimation of the fractal dimension exhibits some inherent uncertainty due to the fact that we only use a sample of points of the object, as well as due to the incomplete accuracy of the numerical algorithms for fractal dimension. For this reason, a new definition of the fractal dimension is being proposed, incorporating the concept of intuitionistic fuzzy sets, which we consider to better capture the uncertainty of the concept.
\end{abstract} Keywords: Fractal dimension, Geometrical complexity, Fuzzy sets, Intuitionistic fuzzy sets. 2010 Mathematics Subject Classification: 03E72, 28A80, 91Gxx.

\section{Introduction}

Traditionally, we would assign a particular geometrical object a crisp value of the fractal dimension, and this numerical value was considered as a specific label for the object. In simple terms, the fractal dimension measures how complex is an object, which can be from nature (like a bacteria) or a mathematical object produced with a formula or an algorithm (like the wellknown Mandelbrot set). However, such numerical values are hard to use in classification or recognition applications since calculated values do not match these crisp values. This problem 
has been observed when implementing the idea for classifying bacteria using the fractal dimension $[3,11]$. While particular numerical labels for each of the bacteria have been found, the attempt to use these values for recognizing specific bacteria in samples has indicated problems due to the presence of uncertainty. Therefrom, the following scheme has been proposed for estimating the fuzzy fractal dimension of a set of geometrical objects. First, the standard fractal dimension of the objects is calculated, using the box counting algorithm with samples of points from the objects. Second, the crisp values for the fractal dimensions of the objects are used to build linguistic values for the dimensions, namely, the fuzzy fractal dimensions of the objects. Third, using these linguistic values of the fractal dimensions, a set of fuzzy rules is built so that each object is relates with each rule. Thus, this set of fuzzy if-then rules can be regarded as a classification scheme for the set of geometrical objects, and can be used for recognizing these objects because a particular value is mapped to an object. This method can be applied either to pattern recognition or to time series analysis as shown in [6]. Initially, using the fractal dimension we need to build the specific classification rules for the considered application. Later, we need to implement an object sampling method in order to obtain the data needed to calculate the crisp value of the fractal dimension. At the end, using this crisp value as input in the set of fuzzy rules, we obtain as output the specific classification for the object. For real image processing, for example robotic applications [4], this can be used for recognition of particular objects. In time series analysis, this can be used for the purpose of modeling and forecasting. In any case, the generalization of the mathematical concept of the fractal dimension [10], to include ideas of fuzzy [19] and intuitionistic fuzzy logic is also significant from theoretical point of view because is only the initial point in the fuzzy and intuitionistic fuzzy generalization of the fractal theory.

The rest of the paper is organized as follows. Section 2 outlines the mathematical definition of the fractal dimension. Section 3 describes the use of fuzzy logic in classification. Section 4 outlines the use of the intuitionistic fuzzy logic in estimating the fractal dimension. Section 5 presents the fuzzy fractal approach for time series analysis. Finally, Section 6 offers the conclusions of this work and outlines possible future work.

\section{Fractal dimension of an object}

In the last decades, there has been notable progress has in understanding the complexity of an object using fractal concepts [10] and dynamic scaling theory. For instance, financial time series exhibit scaled properties, thus suggesting a fractal structure $[2,4]$. The fractal dimension of a geometrical object can be defined as follows:

$$
d=\lim _{r \rightarrow 0} \frac{\ln N(r)}{\ln \left(\frac{1}{r}\right)}
$$

where $N(r)$ denotes the number of boxes covering the object and $r$ denotes the size of the box. An approximation to the fractal dimension can be obtained using the box counting algorithm, i.e., by counting the number of boxes covering the boundary of the object for different $r$ sizes and subsequently performing a logarithmic regression to obtain $d$. In Figure 1, the box counting algorithm is illustrated for a hypothetical curve $C$. Counting the number of boxes for different 
sizes of $r$ and performing a logarithmic linear regression, we can estimate the box dimension of a geometrical object with the following equation:

$$
\ln N(r)=\ln \beta-d \ln r
$$

Illustration of this algorithm is given in Figure 2.

The fractal dimension can be used to characterize any arbitrary object. The explanation is that the fractal dimension evaluates the geometrical complexity of objects. In this case, a time series can be classified by using the numeric value of the fractal dimension ( $d$ is between 1 and 2 since we are on the plane $x y$ ). The reasoning behind this classification scheme is that in case of smooth boundary the fractal dimension of the object will approach 1. Otherwise, in case of a rougher boundary, the fractal dimension will approach 2 .

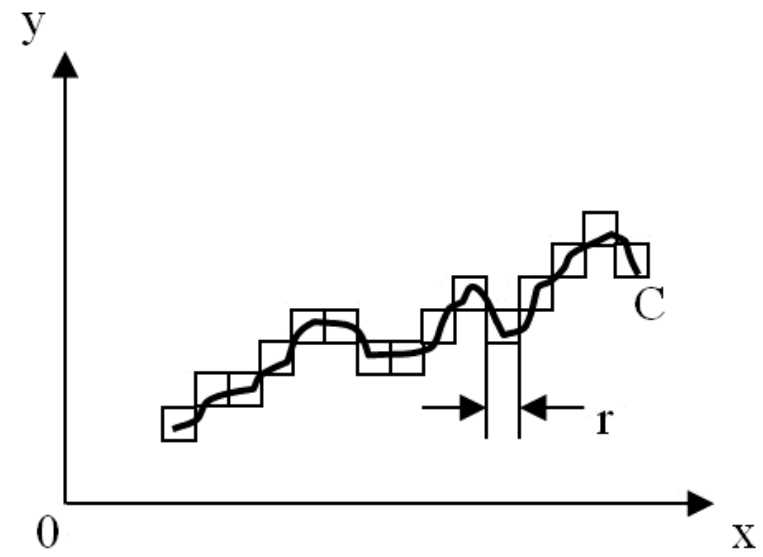

Figure 1. Box counting algorithm for a curve $C$

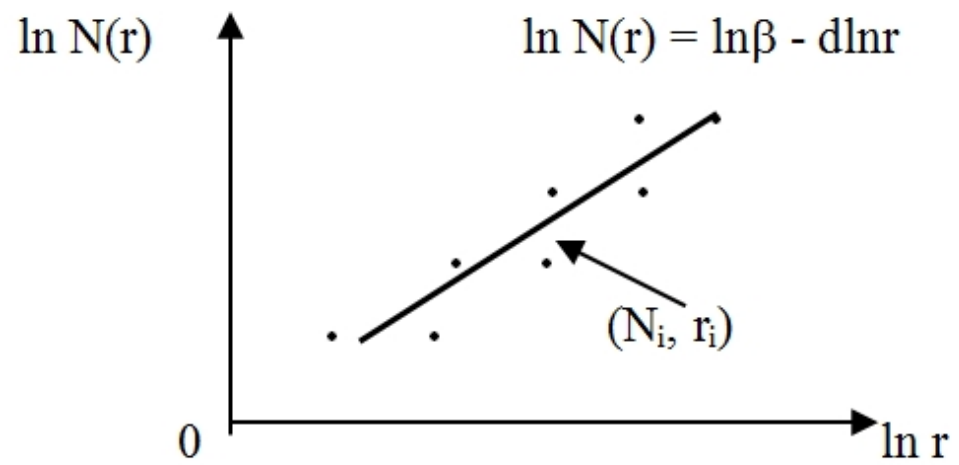

Figure 2. Logarithmic regression to estimate fractal dimension

\section{Fuzzy logic for object classification}

We can use a fuzzy rule base as a classification scheme if we can make a suitable partition of the input space that enables us to differentiate geometrical objects based on their characteristics. For the sake of simplicity, we will consider geometrical objects in the plane. Now we can apply to 
the data fuzzy clustering techniques [2, 18], and then construct a fuzzy rule base that will represent the classification scheme for the specific application.

Let us have $n$ objects $O_{1}, O_{2}, \ldots, O_{n}$, and let us be able to apply fuzzy clustering techniques to obtain $n$ pairs $\left(X_{i}, Y_{i}\right) i=1, \ldots, n$, which represent the respective centers of the $n$ clusters. Hence, a fuzzy rule base can be built as follows:

$$
\begin{aligned}
& \text { If } X \text { is } x_{1} \text { and } Y \text { is } y_{1} \text {, then Object is } O_{1} \\
& \text { If } X \text { is } x_{2} \text { and } Y \text { is } y_{2} \text {, then Object is } O_{2} \\
& \ldots \\
& \text { If } X \text { is } x_{n} \text { and } Y \text { is } y_{n} \text {, then Object is } O_{n}
\end{aligned}
$$

These rules can be utilized for pattern classification or time series analysis since in either case the data exhibits the same general structure. For applications of higher dimensionality, this approach can be generalized in a straightforward manner, but at the expense of dramatic increase of the number of rules (also known as the "curse of dimensionality").

\section{Intuitionistic fuzzy estimation of the fractal dimension}

Intuitionistic Fuzzy Logic was originally proposed by Atanassov [1] and its application here can help modelling the inherent uncertainty in the fractal dimension concept [10] as it is outlined in the proposed approach presented below, which is inspired in an initial proposal of Castillo et al. in [6].

As we noted above, the fractal dimension of a geometrical object is a crisp numerical value measuring the geometrical complexity of the object. However, in practical applications it is challenging to assign a unique numerical value to an object. It is more appropriate to assign a range of numerical values in which there exists a membership degree for this object. For this reason, we will assign to an object $O$ a fuzzy set $\mu_{O}$, which measures the membership degree for that object.

Let us consider that the object $O$ lies in the plane $x y$, then a suitable membership function is defined as a generalized bell function of the kind:

$$
\mu_{O}=\frac{1}{1+\left|\frac{x-c}{a}\right|^{2 b}}
$$

and the non-membership function is expressed as

$$
v_{O}=1-\mu_{O}-\pi_{O}
$$

where $a, b$ and $c$ are the parameters of the membership function, and $\pi$ is known as the hesitation margin [1]. Other types of membership functions can also be utilized with respect to the specifics of the application. In fact, by using the concept of a fuzzy set we are generalizing the mathematical concept of the fractal dimension.

Definition 1: Let $O$ be an arbitrary geometrical object in the plane $x y$. Then the fuzzy fractal dimension is the pair: $\left(d_{O}, \mu_{O}, v_{o}\right)$ where $d_{O}$ denotes the numerical value of the fractal dimension as computed by the box counting algorithm, and $\mu_{O}$ denotes the membership function for the 
object and $v_{o}$ denotes the non-membership function. With this new definition we aim to render a better account for the uncertainty in the estimation of the fractal dimension of an object.

\section{$5 \quad$ Intuitionistic fuzzy fractal approach for time series analysis}

Let us consider now the problem of time series analysis and prediction. Let $y_{1}, y_{2}, \ldots, y_{n}$ be an arbitrary time series. In order to forecast this time series, we have to analyze the data and extract the trends and periodicities of the series. Assuming that the time series can be clustered into $n$ objects $O_{1}, O_{2}, \ldots, O_{n}$ as demonstrated in Figure 3, then we can construct a fuzzy rule base as the one in Section 3 above. The basic idea here is that the complexity of the time series data can be measured with the fractal dimension, and the uncertainty in its value can be handled with intuitionistic fuzzy concepts. However, we now also want to consider the geometrical complexity of the objects $O_{1}, O_{2}, \ldots, O_{n}$ as measured by their fractal dimensions $d_{o_{1}}, d_{o_{2}}, \ldots, d_{o_{n}}$, respectively. Then a fuzzy rule base for time series prediction can be expressed as follows.

If $\operatorname{dim}$ is $d_{O_{1}}$ and pos is $x_{1}$, then prediction is $O_{1}$

If $\operatorname{dim}$ is $d_{\mathrm{O}_{2}}$ and pos is $x_{2}$, then prediction is $\mathrm{O}_{2}$

$\begin{array}{ll}\cdots & \ldots\end{array}$

If $\operatorname{dim}$ is $d_{O_{n}}$ and pos is $x_{n}$, then prediction is $O_{n}$

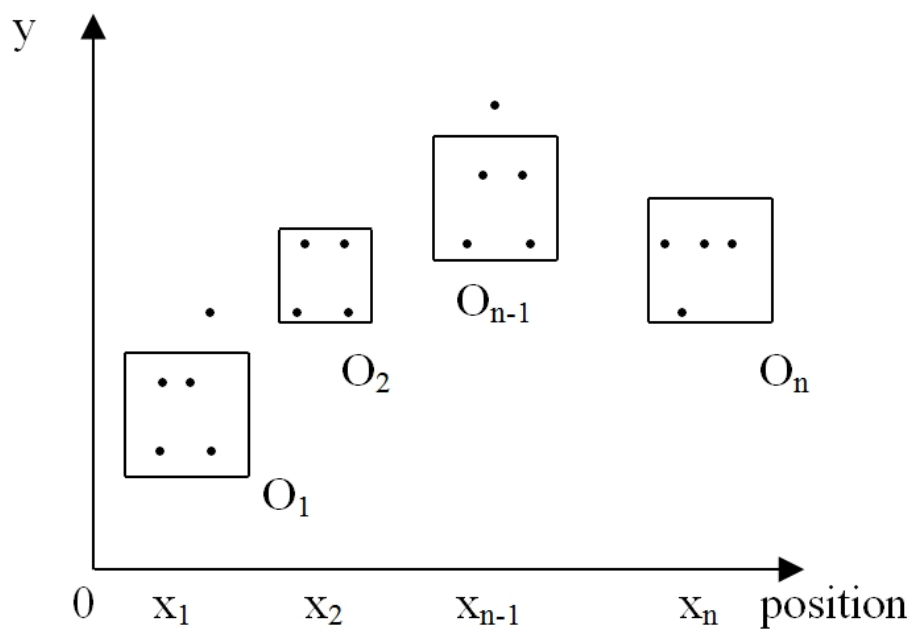

Figure 3. Fuzzy clustering of the time series.

In this case, we need to determine the membership functions for the fractal dimension, position, and the geometrical objects. For the fuzzy rule base Mamdani reasoning method can be used, and center of gravity can be applied as defuzzification method. However, Sugeno type fuzzy system is also possible with linear functions in the consequents. This is illustrated in Equation (7). 
If $\operatorname{dim}$ is $d_{O_{1}}$ and pos is $x_{1}$, then $y=a_{1} x_{1}+b_{1} d_{O_{1}}+c_{1}$

If $\operatorname{dim}$ is $d_{\mathrm{O}_{2}}$ and pos is $x_{2}$, then $y=a_{2} x_{2}+b_{2} d_{O_{2}}+c_{2}$

If $\operatorname{dim}$ is $d_{O_{n}}$ and pos is $x_{n}$, then $y=a_{n} x_{n}+b_{n} d_{O_{n}}+c_{n}$

In this case, a neuro-fuzzy approach can be applied for adapting the parameters of the fuzzy system. For instance, we can use the neuro-fuzzy approach $[12,14]$ to learn from the real data of the problem which are the best values for the coefficients of the linear functions as well as for the membership functions.

\section{Conclusions}

In continuation of [6], we are presenting here a generalization of the fractal dimension concept by the use of intuitionistic fuzzy logic with the aim of modeling the uncertainty of estimating the fractal dimension with real values. We are also presenting an approach for time series analysis, classification and pattern recognition combining fuzzy logic and fractal theory, with ideas of its further development using intuitionistic fuzzy logic. With the presented approach, we propose construction of a set of intuitionistic fuzzy rules using the fractal dimension of the objects to solve the problem of forecasting or recognition. As this work is in initial stages, we envision as future work testing the proposed new intuitionistic fuzzy fractal dimension in real world problems, like in plant monitoring [7], image processing [8, 9], in quality control [14], intelligent control [12], parameter adaptation in meta-heuristics [15] and others [5, 13, 16, 17].

\section{References}

[1] Atanassov, K. (2017). Intuitionistic Fuzzy Logics, Springer, Cham, Switzerland.

[2] Bezdek, J. C. (1981). Pattern Recognition with Fuzzy Objective Function Algorithms, Plenum Press.

[3] Castillo, O., \& Melin, P. (1994). Developing a new method for the identification of microorganisms for the food industry using the fractal dimension, Journal of Fractals, $2(3), 457-460$.

[4] Castillo, O., \& Melin, P. (1998). A new fuzzy-fractal-genetic method for automated mathematical modelling and simulation of robotic dynamic systems, Proc. of FUZZ'98, IEEE Press, Alaska, USA, Vol. 2, 1182-1187.

[5] Castillo, O., \& Melin, P. (1999). A new Fuzzy Inference System for Reasoning with Multiple Differential Equations for Modelling Complex Dynamical Systems, Proc. of CIMCA'99, IOS Press, Vienna, 224-229.

[6] Castillo, O., \& Melin, P. (2000). A new method for fuzzy estimation of the fractal dimension and its applications to time series analysis and pattern recognition, Proc. of NAFIPS'2000, Atlanta, GA, USA. 451-455. 
[7] Castillo, O., \& Melin P. (2004). A new approach for plant monitoring using type-2 fuzzy logic and fractal theory, Int. J. General Systems, 33(2-3), 305-319.

[8] González, C. I., Melin, P., Castro, J. R., Castillo, O., \& Mendoza, O. (2016). Optimization of interval type-2 fuzzy systems for image edge detection, Appl. Soft Computing, 47, 631643.

[9] González, C. I., Melin, P., Castro, J. R., Mendoza, O., \& Castillo, O. (2016). An improved sobel edge detection method based on generalized type-2 fuzzy logic, Soft Computing, 20 (2), 773-784.

[10] Mandelbrot, B. (1987). The Fractal Geometry of Nature, W.H. Freeman and Company.

[11] Melin, P., \& Castillo, O. (1998). An adaptive model-based neuro-fuzzy-fractal controller for biochemical reactors in the food industry, Proceedings of IJCNN'98, IEEE Computer Society Press, Alaska, USA, Vol. 1, 106-111.

[12] Melin, P., \& Castillo O. (2003). Adaptive intelligent control of aircraft systems with a hybrid approach combining neural networks, fuzzy logic and fractal theory, Appl. Soft Comput., 3(4), 353-362.

[13] Melin, P., Amezcua, J., Valdez, F., \& Castillo, O. (2014). A new neural network model based on the LVQ algorithm for multi-class classification of arrhythmias, Inf. Sci. 279, 483-497.

[14] Melin,P., \& Castillo O. (2007). An intelligent hybrid approach for industrial quality control combining neural networks, fuzzy logic and fractal theory, Inf. Sci., 177 (7), 1543-1557.

[15] Olivas, F., Valdez, F., Castillo, O., González, C. I., Martinez, G. E., \& Melin, P. (2017): Ant colony optimization with dynamic parameter adaptation based on interval type-2 fuzzy logic systems. Appl. Soft Comput., 53, 74-87.

[16] Ontiveros, E., Melin, P., Castillo O. (2018). High order $\alpha$-planes integration: A new approach to computational cost reduction of general type-2 fuzzy systems. Eng. Appl. of AI, 74, 186197.

[17] Sánchez, D., \& Melin, P. (2014). Optimization of modular granular neural networks using hierarchical genetic algorithms for human recognition using the ear biometric measure, Eng. Appl. of AI, 27, 41-56.

[18] Yager, R., \& Filev, D. (1994). Generation of fuzzy rules by mountain clustering, Intelligent and Fuzzy Systems, 2 (3), 209-219.

[19] Zadeh, L. A. (1975). The concept of a linguistic variable and its application to approximate reasoning, Information Sciences, 8, 43-80. 